International Journal of Biology, Pharmaey and Allied Sciences (IJBPAS)

'A Bridge Betueen Caboratory and QRendo'

WwW.iibpas.com

\title{
IMPORTANCE OF BACTERIAL EXOPOLYSACCHARIDES: A
}

\section{REVIEW}

\section{YADAV $\mathrm{N}^{1}$, UPADHAYAY $\mathrm{D}^{2}$, MARCHAWALA $\mathrm{F}^{2}$, BHATTACHARYA $\mathrm{I}^{2}$ AND ANDHARE P $^{2 *}$}

1: Student, M. Sc. Microbiology, Parul Institute of Applied Sciences, Parul University, Post Limda, Waghodia, Gujarat, 391760

2: Assistant Professor, Parul Institute of Applied Sciences, Parul University, Post Limda, Waghodia, Gujarat, 391760

*Corresponding Author: E Mail: Dr. Prasad Andhare: prasad.andhare82145@paruluniversity.ac.in; Tel: $+918200614350$

Received 19 ${ }^{\text {th }}$ Jan. 2021; Revised 20 ${ }^{\text {th }}$ Feb. 2021; Accepted $19^{\text {th }}$ March 2021; Available online $1^{\text {st }}$ April 2021 https://doi.org/10.31032/IJBPAS/2021/10.4.1012

ABSTRACT

The ability to produce exopolysaccharides (EPS) is common among lactic acid bacteria (LAB), though the physiological role of these molecules is still unknown. When extended with an inoculation loop in cultures that form long strands, some EPS confer a "ropy" character on LAB, which can be detected. When EPS is formed in situ during milk fermentation, they may act as natural biothickener, giving the product adequate consistency, improving viscosity, and reducing syneresis. Furthermore, some of these EPSs may have health-promoting properties. Increased consumer demand for novel dairy products necessitates a better understanding of the effects of EPS on existing products as well as the quest for new strains that produce EPS with desirable properties. In addition, genetically modified organisms capable of producing high levels of EPS or newly engineered biopolymers are seldom used. Exploration of the heterogeneity of wild LAB strains in natural ecological settings is currently the most powerful method for identifying the target EPS phenotype. In recent years, a variety of techniques have been used to screen ropy strains as well as isolate and characterise the EPS responsible for this characteristic. The information available on the methods and techniques used to conduct research on this subject is outlined 
in this study. The information provided covers LAB-producing EPS screening methods, ropine phenotype detection, and physicochemical and structural characterization of these molecules, including parameters related to their viscosifying properties. To our knowledge, this is the first set of LAB-developed methods for EPS analysis that is publicly accessible.

Keywords: Exopolysaccharides (EPS), Lactic acid bacteria (LAB), halobacteria, methanogens

\section{INTRODUCTION}

Exopolysaccharides are produced by a variety of bacteria, algae, fungi, and yeasts (EPS). Microorganisms' ability to generate EPS is a clear and rational response to selective pressures in the natural world [1]. Microorganisms from hydrothermal vent bacteria, halobacteria, methanogens, autotrophic organisms, acidophils, groundwater, and sewage sludge are examined for exopolysaccharides. Some microorganisms isolated from extreme conditions, such as deep-sea hydrothermal vents, Antarctic ecosystems, saline lakes, and geothermal springs, have begun to be researched as potential sources of useful biopolymers, including EPSs [2]. The physical properties involved in the rheology of solution exopolysaccharides, as well as the ability to form gel at low concentrations, are critical for commercial application of microbial exopolysaccharide. The relevance of bacterial biopolymers to humans has been discovered thanks to technological advancements [3]. Microbial polysaccharides are biodegradable in nature and have a lower environmental impact than synthetic polymers. This adds to their environmental friendliness in manufacturing applications, waste treatment, and other environmental applications [1].

The ability to generate polysaccharides is widespread among bacteria. Storage polysaccharides, such as glycogen in the cytoplasm, structural polysaccharides of the cell wall, such as peptidoglycan and grampositive bacterial lipoteichoic acids, and lipopolysaccharides anchored in the gramnegative bacterial outer membrane can all be synthesised by microorganisms. Furthermore, some bacteria may secrete polysaccharide layers on their surface, which are grouped together with a few glycoproteins as 'glycocalyx.' [4]. Capsular polysaccharides, which form a cohesive sheet or capsule covalently attached to the cell surface, and exopolysaccharides (EPS), which form a slime layer loosely attached to or secreted into the atmosphere by the cell surface, make up these exocellular polymers [5]. Although it is generally recognised that the producer microorganism does not use exocellular polysaccharides as a source of energy or carbon, the physiological function of these molecules remains uncertain [4]. 


\section{EPSS: CHARACTERISTICS AND PHYSIOLOGICAL ROLES:}

Exopolysaccharides (EPSs) are highmolecular polymers made up of sugar residues that microorganisms secrete into the environment. They make up a large portion of the extracellular polymers covering most microbial cells in extreme environments including Antarctic habitats, saline lakes, geothermal springs, and deepsea hydrothermal vents. Extremophiles have developed a range of adaptation methods to compensate for the harmful effects of extreme conditions, such as high temperatures and salt concentrations, low $\mathrm{pH}$ or temperature, and high radiation. EPS biosynthesis is one of the most common protective mechanisms among these methods. In their natural environment, most bacteria live in microbial aggregates, whose structural and functional stability is dependent on the presence of an extracellular polymer material matrix, and the production of EPS appears to be important for their survival [7]. Many microorganisms have been found in extracellular polysaccharides (many species of Gram-positive and Gram-negative bacteria, archaea, fungi and some algae). Sutherland [8] coined the term exopolysaccharide to describe polymers produced by marine bacteria with a high molecular weight of carbohydrates. Exopolysaccharides can be found in the environment as capsular material or as a distributed slime with no obvious link to any particular cell [8]. In the discovery and development of novel industrially important microbial EPSs (Nicolaus et al. 2010), There has been considerable change. Over the last few decades, a large number of microbial EPSs have been discovered, and their composition, structure, biosynthesis, and functional properties have all been thoroughly investigated [9]. Increased demand for natural polymers for pharmaceutical, food, and other industrial applications has sparked interest in microorganism-produced polysaccharides in recent years. Indeed, the isolation and identification of new microbial polysaccharides with novel applications such as gelling, emulsifying, and stabilising agents has piqued interest $[\mathbf{9}, \mathbf{1 0}]$.

Exopolysaccharides are produced by many microorganisms as a strategy for growing, adhering to solid surfaces, and surviving adverse conditions. The ecological niches in which microorganisms have been isolated, as well as the natural environment, affect the physiological role of EPS [10]. Indeed, the production of EPS requires a significant amount of energy, up to $70 \%$ of the total energy reserve, which is a significant carbon expenditure for microorganisms. However, the existence of certain glycosidic linkages and their accessibility to glycolytic enzymes is thought to be 
required for EPS's presumed prebiotic effect. The development of EPS engineering strategies for specific food applications will enable the main parameters involved in EPS functionality to be elucidated. EPSs are unquestionably defensive: they form a layer of surrounding cells that provides effective protection against high or low temperatures, salinity, or possible predators. They are needed for the formation of aggregates, the adhesion process to surfaces and other organisms, biofilm formation, and nutrient absorption [11]. Studies of microbial sea ice populations, in particular, have identified bacteria that are strongly particle-associated and shown that microbial EPSs play an important role in cryoprotection [12].

\section{ROLE OF MICROBIAL EPS}

Microbial polymers were first used in the 1960s, and their commercial use has expanded significantly since then. Microbial EPS plays an important role in cellular interactions, nutrition, and microand macro environments.In the absence of EPS, organisms that produce EPS survive better in oligotrophic environments and live below the nutrient concentration threshold required. They're used in the food, clothing, detergent, beverage, pharmaceutical, biotechnology, forestry, paper, paint, and petroleum industries, as well as drug delivery and cancer care. They're also used in the development of cultural media [13].
Exopolysaccharides (EPS) formed by lactic acid bacteria (LAB) play an important role in the processing of fermented dairy products such as yoghurt, drinking yoghurt, cheese, fermented cream, and milk-based desserts [14]. There is a lot of variation in EPS production by LAB in terms of quantity, chemical composition, molecular size, charge, presence of side chains, and rigidity of the molecules. One of the most important sensory qualities for consumer preference in dairy products is firmness and creaminess.EPS can serve as texturizers and stabilisers to boost the rigidity of the casein network, first by increasing the viscosity of the final product, and then by binding hydration water and interacting with proteins and micelles of milk constituents [15]. As a consequence, EPS will reduce the production of hazardous byproducts while also improving product stability. Furthermore, it has been known that EPS may have a positive effect on gut health. Exopolysaccharide-producing $S$. thermophiles [16] can improve the functional properties of Mozzarella cheese, but they are not phage-proof. The consistency of processed foods is improved by exopolysaccharides. They communicate with water molecules and regulate the rheological and physical properties of foods [1].

Nutraceutical product development has provided the food industry with yet another 
exciting opportunity. Aside from clear control of the active ingredients in the product, the most pressing issue in nutraceutical products is efficacy preservation. The animal feed industry takes advantage of EPS's high suspension and stability properties to transport liquid feed containing vitamins and other nutrients that would otherwise settle during transportation or storage [17].

Indeed, bacterial polysaccharides have been shown to have cation binding and ion absorption properties, enhancing their function in bioremediation processes. The acetylation stage affects the selectivity of certain EPS for metal binding sites [18]. These characteristics could be extremely useful in sewage treatment, especially for the removal of toxic heavy metal contaminants [19]. They have a greater potential for metal complexing, so they can be used in addition to or instead of the conventional metal removal process in mining and industrial waste.Mineral solubilization is found to have a substantial impact in the presence of an EPS layer. Acidithiobacillus ferrooxidans also produces an EPS sheet, which aids metal extraction from sulphidic minerals [20]. Bacteria containing exopolysaccharide have also been used as a bioinoculant to improve the soil rhizosphere's aggregation and water retention capacity as a function of soil water quality. It has been demonstrated that two or more chemically distinct exopolymers can co-synthesize in microorganisms such as Acetobacter sp., Rhizobium sp., Agrobacterium sp., and Alkaligenes sp. These include low molecular weight exopolysaccharides (LMWEPS), such as the b 1-2 glucans. It is also probable that, in reaction to a nearby surface, these additional polymers are synthesised and secreted. Consequently, the development of high molecular weight EPS is manifested in subsequent events such as stabilisation and colony persistence [21]. Rhizobium meliloti's successful invasion of alfalfa relies on both high and low molecular weight EPS development in the rhizosphere ecosystem [22].

\section{LACTOBACILLUS AS AN EPS PRODUCER:}

Exopolysaccharides are repeatable instruments that point to a large class of biotechnological performance polymer materials with a broad variety of applications [23]. Long-chain polysaccharides containing branched, repeating sugar units or sugar derivatives such as glucose, fructose, mannose, galactose, and others are secreted into the environment by bacteria during their development. In addition, microbial exopolysaccharides such as dextrans, xanthan, gellan, pullulan, yeast glucans, and bacterial alginates, such as Xanthomonas campestris xanthan gum and Pseudomonas elodegellan, can be used as 
food additives in a variety of industries [24]. Microorganisms are better suited to the development of EPS than macroalgae and higher plants because they grow rapidly and take advantage of their surroundings, resulting in more microbial EPS [25]. LAB have gained special attention from a wide range of polysaccharide-producing microorganisms due to the remarkable properties of the polymers they synthesise and the fact that they are GRAS (no health risk). In order to enhance probiotic bacteria colonisation, EPS from LAB must stay stable in the gastrointestinal tract. LAB renders EPS in the form of capsular polysaccharides (CPSs) or slime polysaccharides. Slime EPS is secreted into the ambient environment, while CPSs bind heavily to the surface of bacterial cells. These biopolymers are made up of one form of monosaccharide (homopolysaccharides) or repeat units of monosaccharides with different molecular weights (HePSs) ranging from 4.0 to 104 to 6.0 to $106 \mathrm{Da}$ [26].

\section{HOMO EPS BIOSYNTHESIS}

Outside the cell, specific glycosyltransferase (GTF) or fructosyltransferase (FTF) enzymes produce homo EPS (commonly named glucansucrases or fructan-sucrases). Extracellular GTF enzymes are also used by the LAB developing Homo-EPS to synthesise high-molecular-mass alphaglucans from sucrose.This process uses sucrose as a basic substrate, and the energy needed for the process comes from sucrose hydrolysis. There is no energy requirement for EPS production other than for enzyme biosynthesis because EPS synthesis by GTF or FTF does not involve active transport processes or the use of activated carbohydrate precursors. As a result, large quantities of sucrose can be quickly transformed to EPS. In the presence of 160 $\mathrm{g} / 1$ sucrose, Lb. sanfranciscensis produces up to $40 \mathrm{~g} / 1$ levan and $25 \mathrm{~g} / 1$ 1-kestose during development[27].

\section{HETERO EPS BIOSYNTHESIS}

Extracellular enzymes are not used to make hetero-exopolysaccharides; instead, a complex sequence of intracellular enzyme interactions are used. Exopolysaccharides are generated by the polymerization of repeating units, which are created by a sequence of sugar nucleotides added to the cytoplasmic membrane. The sugars are the building blocks for the synthesis process. Various monosaccharides and disaccharides can be used as energy sources by LAB strains via certain well-studied sugar uptake systems, including primary transport systems, direct coupling of sugar translocation to ATP hydrolysis through transport-specific ATPase; secondary sugar carrier systems, coupling of sugar transport to the transport of ions or other solutes, 
both as symport and antiport transport systems; and group translocation systems, coupling of sugar transport to phosphorylation by the phosphoenolpyruvate (PEP)-dependent phosphotransferase system [28]. The unit is sent out through the cell membrane after synthesizing a repeating heteroexopolysaccharides unit and becomes polymerized into the ultimate heteroexopolysaccharides. Therefore, multiple enzymes and proteins are involved in the biosynthesis and secretion of hetero type exopolysaccharides, and the enzymes and proteins involved in these processes may not be inimitable to hetero-EPS anabolism.

\section{ENGINEERING OF BACTERIAL POLYSACCHARIDES: GENERAL STRATEGIES}

Bacterial polysaccharides have diverse unique properties for food applications and are used as viscosifiers, stabilizers, emulsifiers, or gelling agents. Due to these valuable properties several studies were performed to genetically engineer the producing organisms in order to generate novel polysaccharide variants and to improve production. Putative targets for engineering are the molecular weight, composition and sequence of co-monomers as well as addition of substituents. Bacterial polysaccharides by their diversity inherently exhibit a tremendous design space toward the production of new valuable materials [29].

Within the last years intensive research focused on providing insight into the mechanisms underlying bacterial exopolysaccharide biosynthesis pathways. High through-put genome sequencing, functional genomics, protein structure analysis and new bioinformatics tools aid toward identifying new EPS biosynthesis pathways and to understand the principles of EPS formation. Depending on the purpose, engineering strategies can be subdivided into different categories. One goal of EPS production engineering is an increased volumetric productivity to cost effectively produce the various EPS. These studies were mostly aiming at increasing the pool of sugar nucleotides (i.e., EPS precursors) to enhance the carbon flux toward the final polymer. In particular genes of precursor biosynthesis were overexpressed. This strategy was demonstrated to be successful for some EPS producers, but failed in some cases [30]. Additionally, in some cases the overexpression of genes involved in the EPS assembly (e.g., GTs, Wzx, Wzy) resulted in enhanced yields and precursor conversion rates while in other cases it had a negative effect presumably due to distorting the multidomain protein complex involved in polymerization and secretion [31]. Additionally the targeted engineering 
of regulatory proteins could increase productivity by increasing transcription of the operons, which encode the EPS biosynthesis proteins. Furthermore, the disruption of pathways competing for precursors used for EPS formation did also increase the productivity [32]. Single gene knock-outs were also described to enhance yield as well as to alter the chemical structure of the EPS [33]. Unfortunately, the titer of bacterial polysaccharides is limited in the production because the highly viscous polysaccharides have a massive negative influence on mass transfer. However, the strategy to enhance productivity based on genetic engineering might be interesting for EPS with reduced viscosifying properties, for example due to lower molecular weight. The optimization of manufacturing process parameters might be more promising than engineering EPS biosynthesis for many established industrial EPS producers. The highest titers of highly viscous EPS such as xanthan are around 30-50 $\mathrm{g} / \mathrm{L}$ and represent the current maximum amount, which is manageable by existing bioprocess units. Another strategy of engineering EPS biosynthesis is aiming at tailor-made variants with desirable material properties for medical and industrial applications. Here the aim is to alter the molecular structure and therefore the behaviour and material characteristics of the final polymer. For example, these modifications can be based on deleting substituents or monomeric sugars from the side chain. On the other hand, new or more substituents might be attached to change the ratio of decoration. Most efforts were done in engineering the degree of acetylation and pyruvylation of various polymers, in order to control their rheological behavior. Additionally, an altered degree of pyruvylation results in varied charge density of the polysaccharide. Targeted modification of the molecular weight via overexpression or mutation of genes involved in the polymerization/degradation process (e.g., synthase, Wzy, PCP/lyases, glucosidases) represents another possibility to adjust rheology of the final product and was reported for some EPS [34]. The early engineering approaches of xanthan biosynthesis as performed by Hassler and Doherty (1990)gave interesting insights in general structure-function relationships. The strongest influence on rheology was observed by altering the substituent decoration. The degree of acetylation and pyruvylation has opposite effects on viscosity. A high degree of pyruvylation resulted in higher viscosity, whereas more acetyl groups decreased viscosity of the resulting EPS. This finding is a general rule for polysaccharides and can be used in tailoring the viscosity of EPS. The degree of acetylation can be adjusted by in vivo as 
well as in vitro approaches or even process parameters applied during the production process [35]. Further engineering approaches with respect to the production of xanthan variants included the targeted engineering of the length of the side chain. These approaches are of great interest, since they might be transferred to other EPS variant.The influence of acetylation on viscosity of the truncated versions showed an irregular result. The acetylated polytetramer version showed decreased viscosity as observed for the wild type xanthan. The acetylation of the polytrimer (only one mannose as side chain) resulted in similar viscosity as the non-acetylated version. Pyruvylation of the outer mannose also blocks acetylation of this sugar, therefore enhancing the viscosity.

\section{CONCLUSION}

In summary, complete characterization of the EPS requires information on the phenotype of the microbial EPS, the quantity of EPS formed, the composition of the sugar, the structure and molecular parameters associated with the intensifying viscosity properties. Those using gas and liquid chromatography with various detection methods and nuclear magnetic resonance are the techniques most commonly used for the study of monomer composition, molar mass, gyration radius, and structure. The wide range of cultural media and methods used to isolate and measure EPS tend to have a strong effect on the results of the yield, making it difficult to compare the values obtained by various authors. Few studies compare the degree of purity obtained after isolation in the EPS fraction by the various currently available methods. This fact is of great importance given that for specific studies on chemical composition, structure, and physical characterization of EPS molecules, isolation procedures that yield a high purity EPS fraction are desirable or even required.In spite of this, it is important to note that the advancement of the procedures and analytical methods summarized in this review has allowed a large number of EPS developed by LAB strains to be isolated and characterized from various natural ecological niches in recent years. The task for future LABproduced EPS research is to elucidate the relationship between structure and function. It is understood that the quantity, chemical composition, chain length and molecular size of EPS molecules affect the physical properties of fermented milk products. It is now possible to compare a particular EPS structure with precise texture and viscosity values. In addition, the molecular features of EPS that may play a role in the beneficial effects on human health are not yet well known. However, the presence and accessibility of certain glycosidic linkages to glycolytic enzymes is expected to be 
essential for the putative prebiotic effect of EPS. The creation of EPS engineering strategies for specific food applications will enable the elucidation of the key parameters involved in EPS functionality.

\section{ACKNOWLEDGEMENT}

It is our privilege and honour to express our sincerest gratitude to the Parul University, Vadodara, Gujarat for providing me all the necessary support and facilities including state of the art infrastructural facilities with advanced technological scientific laboratories and everything else that was required to carry out this work.

\section{REFERANCES}

[1] Shailesh R Dave,Avni M Vaishnavet al; Microbial exopolysaccharide - an inevitable product for living beings and environment. J Bacteriol Mycol Open Access. Aug 2016; 2(4):109-111.

[2] Annarita Poli, Paola Di Donatoet al; Synthesis, Production, and Biotechnological Applications of Exopolysaccharides and Polyhydroxyalkanoates by Archaea. Archaea, Oct 2011; 2011:1-13.

[3] Uchechukwu U Nwodo Ezekiel Green et al; Bacterial exopolysaccharides: functionality and prospects. Int J Mol Sci. Oct 2012; 13(11): 14002-14015.

[4] P.Ruas-Madiedo andC.G. de los Reyes-Gavilán. Invited Review:
Methods for the Screening, Isolation, and Characterization of Exopolysaccharides Produced by Lactic Acid Bacteria. J. Dairy Sci. March 2005; 88(3):843-856.

[5] Michael T Madigan,John M Martinkoet al;Brock Biology of Microorganisms. 11th ed. Prentice Hall International Ltd., London, UK, 2006.

[6] Jost Wingender, Thomas R. Neu et al; Microbial extracellular polymeric substances: characterization, structure and function. Germany: Springer Science \& Business Media, Springer-Verlag Berlin Heidelberg, 2012: 258.

[7] Sutherland IW. Novel and established applications of microbial polysaccharides. Trends Biotechnol. 1998; 16(1): 41-46.

[8] Barbara Nicolau, Margarita Kambourova et al; Exopolysaccharides from extremophiles: from fundamentals to biotechnology. Environ Technol. Sep 2010; 31(10): 1145-1158.

[9] Annarita Poli, Paola Di Donato et al; High level synthesis of levan by a novel Halomonas species growing on defined media. Carbohydrate Polymers. Oct 2009; 78(4): 651657. 
[10] Martin Krüger, Martin Blumenberg et al; A novel, multilayered methanotrophic microbial mat system growing on the sediment of the Black Sea. Environmental Microbiology. Aug 2008; 10(8): 1934-1947.

[11] Karen Junge, Hajo Eicken et al; Bacterial activity at -2 to $-20^{\circ} \mathrm{C}$ in Arctic wintertime Sea Ice. Applied and Environmental Microbiology. Jan 2004; 70(1): $550-557$,

[12] Emilia Quesada, Victoria Béjaret al; Exopolysaccharide production by Volcaniella eurihalina. Experientia. Dec 1993; 49(12): 1037-1041.

[13] PhilippeDuboc and BeatMollet. Applications of exopolysaccharides in the dairy industry. International Dairy Journal.Oct 2001; 11(9): 759-768.

[14] Forough Sadat Tabibloghmany and Elham Ehsandoost. An overview of healthy and functionality of exopolysaccharides produced by lactic acid bacteria in the dairy industry. European Journal of Food Research \& Review. June 2014; 4(2): 63-86.

[15] BroadbentJeffery R, McMahon, Donald Jet al; Biochemistry, genetics, and applications of exopolysaccharide production in Streptococcus thermophilus: a review. J Dairy Sci. Feb 2003; 86(2): 407-423.

[16] Gordon Morris and Harding Stephen. Polysaccharides, microbial. Encyclopedia of microbiology. Dec 2009; 3: 482-494.

[17] Ian W Sutherland. Biosynthesis and composition of gram-negative bacterial extracellular and wall polysaccharides. Ann Rev Microbiol. Oct 1985; 39(1): 243270.

[18] Sun Beom Choi and Yeoung-Sang Yun. Biosorption of cadmium by various types of dried sludge:an equilibrium study and investigation of mechanisms. J Hazard Mater. May 2006; 138(2): 378-383.

[19] Yu Runlan and Ou Yang et al; Effect of EPS on adhesion of Acidithiobacillus ferrooxidans on chalcopyrite and pyrite mineral surfaces. Transactions of Nonferrous Metals Society of China. Feb 2011; 21(2): 407-412.
[20] David
G.
Allison.

Exopolysaccharide production in bacterial biofilms. Biofilm. March 1998: 3(1) 
[21] Laurie Battisti, Jimmie C et al; Specific oligosaccharide form of the Rhizobium meliloti exopolysaccharide promotes nodule invasion in alfalfa. Proc Natl Acad Sci USA. Feb 1992; 89(12): 5625-5629.

[22] Anita Suresh Kumar, Kalpana Mody et al; Bacterial polysaccharides - a perception. Journal of Basic Microbiology. April 2007; 47(2): 103-117.

[23] Abbas Abedfar and Marzieh Hosseininezhad. Overview of the most important characterization of exopolysaccharides produced by probiotics bacteria and their biological function. IOSR Journal of Environmental Science, Toxicology and Food Technology (IOSR-JESTFT). Nov 2016; 10(11): 47-55.

[24] Amit Parikh, and Datta M. Partial characterization of extracellular polysaccharides from Cyanobacteria. Bioresource Technology. Oct 2006; 97(15): 1822-1827.

[25] Mozzi F and Savoy de Giori et al; UDP-galactose 4-epimerase: a key enzyme in exopolysaccharide formation by Lactobacillus casei CRL 87 in controlled $\mathrm{pH}$ batch cultures, Journal of Applied
Microbiology. Jan 2003; 94(2): 175-183.

[26] Maher Korakli, Melanie Pavlovic et al; Exopolysaccharide and Kestose Production by Lactobacillus sanfranciscensis LTH 2590. Applied and Environmental Microbiology. April 2003; 69 (4): 2073-2079.

[27] Luc De Vuyst, Filip De Vin et al; Recent developments in the biosynthesis of hetero-polysaccharides of lactic acid bacteria. International Dairy Journal. Oct 2001; 11(9): 687-707.

[28] Jochen Schmid, Volker Sieber et al; Bacterial exopolysaccharides: biosynthesis pathways and engineering strategies. Front. Microbiol. May 2015; 6:496.

[29] L Thorne, MJ Mikolajczak et al; Increasing the yield and viscosity of exopolysaccharides secreted by Sphingomonas by augmentation of chromosomal genes with multiple copies of cloned biosynthetic genes. J. Ind. Microbiol. Biotech. July 2000; 25(1): 49-57.

[30] Richardvan Kranenburg, Ingeborg $\mathrm{C}$ Boels et al; Genetics and engineering of microbial exopolysaccharides for food: approaches for the production of existing and novel polysaccharides. Curr. Opin. 
Biotechnol. Oct 1999; 10(5): 498 504.

[31] C Pena, L Hernandez et al; Manipulation of the acetylation degree of Azotobacter vinelandii alginate by supplementing the culture medium with 3-(Nmorpholino)-propane-sulfonic acid. Lett. Appl. Microbiol. Aug 2006; 43(2): 200- 204.

[32] Cinthia Núñez Alexander V Bogachev et al; The $\mathrm{Na}+$ translocating NADH: ubiquinone oxidoreductase of Azotobacter vinelandii negatively regulates alginate synthesis. Microbiology. Jan 2009; 155(1): 249-256.

[33] Bernd H A Rehm. Bacterial polymers: biosynthesis, modifycations and applications. Nature Rev Microbiol. Aug 2010; 8(8): 578-592.

[34] Bernd H. A. Rehm. Synthetic biology towards the synthesis of custom made polysaccharides. Microb. Biotechnol. Jan 2015; 8(1): 19-20. 